\title{
Entrainment of Neural Activity Using Transcranial Magnetic Stimulation
}

\author{
Dobromir Rahnev \\ Helen Wills Neuroscience Institute, University of California, Berkeley, California 94720 \\ Review of Chanes et al.
}

Transcranial magnetic stimulation (TMS) is one of the most valuable tools at the disposal of cognitive neuroscientists due to its ability to perturb brain function with high temporal precision. To date, TMS has primarily been applied to disturb normal processing nonspecifically, similar to a "virtual lesion." Notwithstanding the creative studies that have allowed researchers to make important progress using this approach, the usefulness of TMS could be greatly increased by developing new applications to modulate specific features of neural processing.

One particularly promising application of TMS relates to entrainment of brain activity at specific frequencies. It has been theorized that neuronal oscillation and synchronization is critical for communication between separate brain regions (Fries, 2005). In a seminal paper, Thut et al. (2011) demonstrated that rhythmic TMS at the alpha frequency $(8-14 \mathrm{~Hz}$, i.e., one pulse every 70-125 ms) could be used to "entrain" brain oscillations in the parietal cortex. Other studies have shown that such alpha-frequency rhythmic TMS affects behavior (Sauseng et al., 2009; Romei et al., 2010, 2011).

Recently, in a paper published in The Journal of Neuroscience, Chanes et al. (2013)

Received May 12, 2013; revised June 5, 2013; accepted June 6, 2013.

I am grateful to lan Cameron, Derek Nee, Justin Riddle, Hakwan Lau, and Sneha Subramanian for helpful comments on this manuscript.

Correspondence should be addressed to Dobromir Rahnev, D'Esposito

Laboratory, University of California, 10 Giannini Hall, Berkeley, CA 94720. E-mail: drahnev@gmail.com.

DOI:10.1523/JNEUROSCI.2012-13.2013

Copyright $\odot 2013$ the authors $\quad 0270-6474 / 13 / 3311325-02 \$ 15.00 / 0$ used TMS to entrain neural oscillations at higher frequencies. The researchers applied four consecutive pulses separated by $34 \mathrm{~ms}$ (a stimulation rate of $30 \mathrm{~Hz}$, or high beta frequency) or $20 \mathrm{~ms}$ (a stimulation rate of 50 $\mathrm{Hz}$, gamma frequency). In addition, the authors used two non-frequency-specific conditions that featured a larger gap between the second and third pulses thus ensuring that no single frequency was preferentially entrained. The non-frequency-specific conditions matched the rhythmic TMS conditions for the number of pulses and the total stimulation duration but not for the rhythm of application.

Chanes and colleagues (2013) stimulated the frontal eye field (FEF), a region involved in eye movement and spatial attention. To investigate the behavioral effects of rhythmic TMS, participants were asked to complete two tasks. In the first task, observers judged whether a briefly presented Gabor patch, a sinewave grating with a Gaussian contrast envelope, was tilted in a clockwise or counterclockwise direction (discrimination task). The Gabor patch could appear left or right of fixation or not appear at all. In the second task, participants were further asked whether they had detected the presence of the Gabor patch, and if so, on which side of the screen it appeared (detection task). Chanes and colleagues used signal detection theory (SDT) to separately investigate the influence of TMS on perceptual sensitivity (quantified by the variable $d$ ' that roughly corresponds to the percentage of correct responses) and response bias (quantified by the variable $\beta$ that roughly corresponds to the percentage of "yes" responses). This use of SDT was needed because $d$ ' and $\beta$ are mathematically independent, unlike the standard measures of percentage correct and the percentage of yes responses that are not independent and therefore are not suitable for experiments in which both performance and subjective bias to respond "yes" are likely affected.

The results revealed selective, frequencydependent effects of TMS application on the detection task. More specifically, the $30 \mathrm{~Hz}$, high-beta-frequency stimulation led to an increase in $d^{\prime}$, whereas the $50 \mathrm{~Hz}$, gammafrequency stimulation led to a decrease in the criterion $\beta$ (i.e., the tendency to respond "yes" more often). The non-frequencyspecific conditions did not result in such effects, thus indicating that these results depend on the frequency of stimulation and not just the total stimulation duration. No significant effects were observed for the discrimination task.

These findings add to a growing literature demonstrating that the effect of TMS extends well beyond the virtual lesion framework. Furthermore, the current results provide evidence against another common misconception: that the strength of the TMS effect depends solely on the intensity of stimulation and the number of pulses. Chanes et al.'s (2013) findings demonstrate that the pattern of TMS pulses influences the TMS effect, even when the number of pulses and intensity of stimulation are held constant.

Nevertheless, the precise mechanisms of 30 and $50 \mathrm{~Hz}$ TMS are still unknown. 
Because TMS was applied to FEF, a central node of the dorsal attention network, it is natural to search for an attention-based interpretation of the results. In particular, it is possible that 30 and $50 \mathrm{~Hz}$ stimulation generated the behavioral effects described above by affecting different kinds of attention. In fact, Chanes et al. (2013) chose these frequencies in part because previous research has linked interareal synchrony in the beta and gamma bands to endogenous and exogenous attention, respectively (Buschman and Miller, 2007). However, several aspects of the data resist the simple interpretation that the effects of the rhythmic TMS in this study are best described as enhancing or suppressing different kinds of attention. For example, only the $30 \mathrm{~Hz}$, and not $50 \mathrm{~Hz}$, condition increased perceptual sensitivity ( $d$ ') but both exogenous and endogenous attention have previously been shown to do so (Carrasco, 2011). In addition, the $d$ ' increase was observed only for the detection, not for the discrimination task, but the latter would also be expected to be affected if TMS acted to boost attention. Last, the $50 \mathrm{~Hz}$ condition induced a more liberal response bias (i.e., an increased tendency to respond "yes"), but recent psychophysics experiments show that spatial attention leads to a conservative, rather than liberal, detection bias (Rahnev et al., 2011; Wyart et al., 2012).

If 30 and $50 \mathrm{~Hz}$ stimulation cannot be mapped neatly onto the constructs of enhancing or suppressing endogenous and exogenous attention, then perhaps one should search for an explanation at a more mechanistic level. For example, the two TMS conditions may have affected differently the communication between FEF and upstream and downstream regions. In that view, different rhythmic stimulations could trigger changes in neural activity in different remote areas in the brain. Conversely, it might be that the effect of TMS did not spread to remote cortical areas but that rhythmic TMS at different frequencies preferentially affected different neuronal populations within FEF (e.g., in different cortical layers). Nevertheless, explanations at this point will remain highly speculative because of our limited knowledge of the neural effects of rhythmic TMS. Therefore, it is critical for future studies to both confirm that 30 and $50 \mathrm{~Hz}$ indeed lead to entrainment of neural activity in the underlying neural tissue and to investigate whether this entrainment spreads to remote regions.

One remaining question is how the present results relate to other proposed mechanisms for the behavioral effect of TMS. For example, prior work has suggested that in certain contexts TMS appears to affect the strength (Harris et al., 2008) or variance (Schwarzkopf et al., 2011) of the perceptual signal. It is important to note that the present results do not necessarily contradict these effects. For example, TMS applied after the stimulus onset might interfere with the neural oscillations critical for stimulus processing thus decreasing signal strength. Similarly, because TMS entrainment interacts with the ongoing neuronal fluctuations (Hamidi et al., 2009; Thut et al., 2011), TMS pulses might lead to better or worse processing in trials in which TMS and ongoing fluctuations are in or out of phase. Across trials, such an interaction could manifest itself as an increase in signal variability. Therefore, neural entrainment does not invalidate previous proposals about TMS affecting signal strength or variability but rather provides a potential approach for understanding the emergence of these effects.

It is also important to consider the relationship between the frequency of neuronal entrainment and the task that subjects engage in. Chanes et al. (2013) observed significant effects of rhythmic TMS on the detection but not the discrimination task. Previous studies that applied rhythmic TMS in the alpha frequency have also found changes in detection tasks (Romei et al., 2010) but discrimination tasks have not been investigated with alpha frequency. It is likely that rhythmic TMS does not apply to both types of tasks in the same way, because detection and discrimination tasks likely depend on fundamentally different computational mechanisms. Indeed, discrimination tasks involve the comparison of the activity of two neural populations in an upstream area by a downstream, decisional area. On the other hand, in detection tasks, only one neural population is involved and the decision is made based on whether the activity in that population reaches a threshold.

Future studies should investigate more systematically the possibility that neuronal entrainment is particularly effective for detection tasks. Such studies may, however, need to separate the detection and discrimination tasks in different conditions. The design used by Chanes et al. (2013) cleverly combines the two task types, but as a result, it may not fit well with the assumptions of SDT that presupposes that the signal and noise distributions are normally distributed. Indeed, because stimuli could appear on either left or right of fixation, two underlying signal distributions were present in the detection task. Chanes and colleagues needed to combine these two distributions into one by excluding trials in which subjects mistakenly identified stimuli from one distribution as belonging to the other, but this process may have resulted in a violation of SDT's normality assumption. Future studies can avoid this issue by presenting all stimuli at fixation or by cuing the side where the stimulus may have appeared and thus making the design more consistent with the SDT assumptions.

\section{References}

Buschman TJ, Miller EK (2007) Top-down versus bottom-up control of attention in the prefrontal and posterior parietal cortices. Science 315:1860-1862. CrossRef Medline

Carrasco M (2011) Visual attention: the past 25 years. Vis Res 51:1484-1525. CrossRef Medline

Chanes L, Quentin R, Tallon-Baudry C, ValeroCabre A (2013) Causal frequency-specific contributions of frontal spatiotemporal patterns induced by non-invasive neurostimulation to human visual performance. J Neurosci 33:5000-5005. CrossRef Medline

Fries P (2005) A mechanism for cognitive dynamics: neuronal communication through neuronal coherence. Trends Cogn Sci 9: 474-480. CrossRef Medline

Hamidi M, Slagter HA, Tononi G, Postle BR (2009) Repetitive transcranial magnetic stimulation affects behavior by biasing endogenous cortical oscillations. Front Integr Neurosci 3:14. CrossRef Medline

Harris JA, Clifford CW, Miniussi C (2008) The functional effect of transcranial magnetic stimulation: signal suppression or neural noise generation? J Cogn Neurosci 20:734-740. CrossRef Medline

Rahnev D, Maniscalco B, Graves T, Huang E, de Lange FP, Lau H (2011) Attention induces conservative subjective biases in visual perception. Nat Neurosci 14:1513-1515. CrossRef Medline

Romei V, Gross J, Thut G (2010) On the role of prestimulus alpha rhythms over occipitoparietal areas in visual input regulation: correlation or causation? J Neurosci 30:8692-8697. CrossRef Medline

Romei V, Driver J, Schyns PG, Thut G (2011) Rhythmic TMS over parietal cortex links distinct brain frequencies to global versus local visual processing. Curr Biol 21:334-337. CrossRef Medline

Sauseng P, Klimesch W, Heise KF, Gruber WR, Holz E, Karim AA, Glennon M, Gerloff C, Birbaumer N, Hummel FC (2009) Brain oscillatory substrates of visual short-term memory capacity. Curr Biol 19:1846-1852. CrossRef Medline

Schwarzkopf DS, Silvanto J, Rees G (2011) Stochastic resonance effects reveal the neural mechanisms of transcranial magnetic stimulation. J Neurosci 31:3143-3147. CrossRef Medline

Thut G, Veniero D, Romei V, Miniussi C, Schyns P, Gross J (2011) Rhythmic TMS causes local entrainment of natural oscillatory signatures. Curr Biol 21:1176-1185. CrossRef Medline

Wyart V, Nobre AC, Summerfield C (2012) Dissociable prior influences of signal probability and relevance on visual contrast sensitivity. 
Proc Natl Acad Sci U S A 109:3593-3598.

CrossRef Medline 